\title{
DOES SENTINEL NODE BIOPSY PLAY A ROLE IN PANCREATIC ADENOCARCINOMA?
}

\author{
Nicolae BACALBASA ${ }^{1,2,3 凶}$, Irina BALESCU ${ }^{4}$, Cristian BALALAU ${ }^{2,5}$, Mihaela VILCU ${ }^{2,3}$, \\ Iulian BREZEAN ${ }^{2,3}$ \\ ${ }^{1}$ Center of Excellence in Translational Medicine, Fundeni Clinical Institute, Bucharest, Romania \\ 2 "Carol Davila“ University of Medicine and Pharmacy, Bucharest, Romania \\ 3 „Ion Cantacuzino“ Clinical Hospital, Bucharest, Romania \\ ${ }^{4}$ Ponderas Academic Hospital, Bucharest, Romania \\ 5 „Sf. Pantelimon“ Clinical Hospital, Bucharest, Romania
}

Received 25 Mar 2019, Accepted 03 May 2019

hitps://doi.org/10.31688/ABMU.2019.54.2.18

\section{Abstract}

Pancreatic adenocarcinoma remains one of the most aggressive malignancies worldwide which is unfortunately associated with very poor long-term survival. However, it seems that the only option in order to improve the outcomes of these patients remains radical surgery consisting of in block resection of the tumor and the surrounding tissues. When it comes to the extent of the lymph node dissection, a permanent debate still exists between the eastern and the western countries; while the Western countries consider that an extended lymph node dissection increases the postoperative complication worthwhile, the Eastern countries consider that this should be the treatment of choice as long as these tumors usually disseminate on the lymphatic route. In order to establish what patients could benefit most from an extended lymph node dissection, certain authors proposed performing the sentinel lymph node biopsy technique. This is a literature review of the largest studies conducted on this theme.

Keywords: sentinel node biopsy, pancreatic adenocarcinoma, lymph node metastases.

\section{Résumé}

La biopsie du ganglion sentinelle joue-telle un rôle dans l'adénocarcinome du pancréas?

Ladénocarcinome du pancréas reste l'une des tumeurs malignes les plus agressives au monde, malheureusement associée à une survie à long terme très médiocre. Cependant, il semble que la seule option pour améliorer les résultats de ces patients reste la chirurgie radicale consistant en une résection en bloc de la tumeur et des tissus environnants. En ce qui concerne l'étendue de la lymphadénectomie, un débat permanent persiste entre les pays de l'Est et de l'Ouest de l'Europe. Alors que les pays occidentaux considèrent qu'une dissection étendue des ganglions lymphatiques accroît la complication postopératoire, les pays orientaux considèrent qu'il devrait s'agir du traitement de choix tant que ces tumeurs se disséminent habituellement par voie lymphatique. Afin de déterminer quels patients pourraient bénéficier le plus après une dissection étendue, certains auteurs ont proposé de réaliser la technique de biopsie du ganglion sentinelle. Il s'agit d'une revue de la littérature des plus grandes études menées sur ce thème. 
Mots-clés: biopsie du ganglion sentinelle, adénocarcinome du pancréas, métastases ganglionnaires.

\section{INTRODUCTION}

Pancreatic adenocarcinoma still represents one of the most fearful malignancies, being associated with an extremely poor survival rate. This aspect is explained through the fact that most patients are diagnosed in advanced stages of the disease, when local invasion and/or distant metastases are already present ${ }^{1}$. Unlike other malignancies, in which the incidence of advanced stage disease and the cancer-related mortality reported a decreasing trend, in pancreatic cancer these parameters remain at an extremely high level, irrespectively to the type of performed therapeutic strategy ${ }^{2}$. In such cases, surgery with curative intent is no longer feasible, so the patient remains a candidate only for palliative treatment. In the remaining cases, in which the disease is diagnosed in a less advanced stage of the disease, it seems that the nodal status has a very important role in regard of the long term outcomes ${ }^{3.7}$.

\section{Pancreatic tumor Resection and lymph nOde DISSECTION IN PANCREATIC ADENOCARCINOMA}

Depending on the location of the primary tumor, certain types of pancreatic resections have been proposed; therefore, for pancreatic head malignancies, the surgical procedure of choice remains pancreatoduodenectomy, procedure performed for the first time by Allen Whipple in $1940^{8}$; during this surgical procedure, the pancreatic head is removed in block with the distal part of the common bile duct, duodenum, gallbladder, as well as with the peri-duodenal and peri-pancreatic lymph node stations ${ }^{8,9}$. In cases presenting central or distal pancreatic tumors, a variety of procedures, such as distal pancreatectomy, alone or in association with splenectomy or central pancreatectomy, have been proposed ${ }^{10-12}$. While the extent of the surgical procedures in terms of visceral resections has been widely accepted, the extent of the lymph node dissection has been considered as a permanent subject of debate. This fact is mainly correlated with the high capacity of the pancreatic adenocarcinoma to spread via lymphatic route; therefore, it has been demonstrated that lymph node metastases develop early in the course of disease in pancreatic adenocarcinomas and represent one of the most important prognostic factors for the long-term survival. However, the extent of the lymph node dissection has been permanently debated, different opinions being reported so far; while the European countries sustain the idea that an extended lymph node dissection in pancreatic adenocarcinoma does not improve the survival and, moreover, increases the postoperative morbidity rates, the Eastern countries consider that such a lymph node dissection is perfectly justified in order to increase the chances for an improved survival ${ }^{13-17}$.

One of the most debated subjects when it comes to the extent of the lymph node dissection is related to the para-aortic lymph nodes; in order to find out which is the chance to have metastatic cells at this level in patients with hepato-biliary-pancreatic malignancies, Noji et al conducted a study on 62 such patients, in whom they injected indocyanine green into the sub-serous membrane in the Calot's triangle ${ }^{18}$. In order to study which is the most appropriate amount of substance to be injected, the authors randomly grouped the 62 cases in three groups: in the first group, $1 \mathrm{mg} / \mathrm{mL}$ of indocyanine green was injected, in the second group $2.5 \mathrm{mg} / \mathrm{mL}$ were injected, while in the third group $5 \mathrm{mg} / \mathrm{mL}$ were used; in all cases, the indocyanine green was diluted in saline solution of a final volume of $0.5 \mathrm{~mL}$; the authors reported a rate of para-aortic node detection of $20 \%$ in cases in which $1 \mathrm{mg} / \mathrm{mL}$ was injected, $80 \%$ for cases in which $2.5 \mathrm{mg} / \mathrm{mL}$ were injected, and $100 \%$ respectively in cases in which a concentration of $5 \mathrm{mg} /$ $\mathrm{mL}$ was used. The authors also demonstrated that the recommended interval of time between injection and performing of the Kocher maneuver should be of 10 minutes in order to maximize the rate of detection of the para-aortic lymph nodes; however, due to the presence of extended adhesions at the level of the hepato-duodenal ligament, the procedure could not be performed in 20 cases. In the remaining 42 cases, in whom the identification of the para-aortic lymph nodes was successfully completed, the pancreatic origin of the tumor was described in five cases. When it comes to the location of the sentinel nodes in these cases, the authors reported their location at the level of the posterior surface of the pancreatic head, as well as on the right border of the abdominal aorta and at the origin of the left renal vein, suggesting that in patients with hepato-biliary-pancreatic carcinomas, injecting $5 \mathrm{mg} / \mathrm{mL}$ of indocyanine green solution might be useful, in order to detect the presence of the sentinel node at the level of the para-aortic region ${ }^{18}$.

\section{Sentinel node detection in Pancreatic Cancer}

Although the anatomical study of the lymphatic system of the pancreas reported a significant 
development, it seems that the use of lymphatic mapping in pancreatic adenocarcinoma still remains limited. It has been stated the fact that lymphatic drainage of the pancreas is a very complex one, presenting multiple lymphatic channels and different lymphatic routes, concentrating the lymphatic outflow in a high number of presumed sentinel nodes, which are mainly located at the level of the retroperitoneal space; therefore, the analysis of these patterns of spread is very difficult ${ }^{19}$. Moreover, it seems that the use of the most representative imagistic methods, such as computed tomography, magnetic resonance or even positron emission tomography, are not reliable in order to offer precise information in regard to the lymphatic status in patients with pancreatic adenocarcinomas $^{20,21}$.

In order to improve the methods of evaluation and detection of the possible lymph node involvement in pancreatic adenocarcinoma, certain authors proposed the use of the sentinel node biopsy.

An interesting study which investigated the efficacy of the method was conducted by Hirono et al and was published in $2012^{22}$. The authors investigated the main patterns of lymphatic spread in 20 patients with pancreatic head adenocarcinomas: in 10 cases, the authors injected the tracer in the anterior surface of the pancreatic head, while in the remaining 10 cases the tracer was injected in the posterior surface. The authors demonstrated that there are seven main lymphatic patterns of spread, the most commonly encountered being the one related to the para-aortic lymph nodes (in $85 \%$ cases) followed by the superior mesenteric artery (in 20\% of cases). Moreover, the authors underlined the fact that the mean interval to reach the lymph nodes at the origin of the superior mesenteric artery was longer to those in para-aortic area, suggesting that the lymph node dissection at the level of the two areas might play a similar oncological role ${ }^{22}$. These data demonstrating the presence of seven main lymphatic routes came to demonstrate once again the complexity of the lymphatic drainage from the pancreatic head.

When it comes to the utility of sentinel node detection in pancreatic body adenocarcinoma, preliminary results published by the Polish authors conducted by Durczynski do not seem very encouraging. The authors included in their study 13 patients with locally advanced pancreatic body tumors submitted to surgery at the Medical University of Lodz during the year of $2010^{23}$; in all cases, $1 \mathrm{~mL}$ of patent blue was injected in the center of the pancreatic tumor just after opening the lesser sac. Five minutes later, the colored lymphatic nodes were retrieved and sent to the histopathological studies. After performing this protocol, the detection rate of the presumptive sentinel node was of only $38.4 \%$, most commonly the sentinel node being located at the level of the proximal area of the splenic artery $(11 \mathrm{p}$ lymph node station). However, the results of the study are discouraging, all the sentinel nodes as well as the retrieved non-sentinel nodes being metastatic ${ }^{22}$. This aspect might be explained through the fact that most cases presented large tumoral masses which are thought to invade and block the lymphatic structures in the close proximity, as well as by the fact that the tracer was injected directly in the center of the tumor. Another explanation given by the authors for these results is related to the learning curve; the authors underlined the fact that in breast cancer surgery, the learning curve for sentinel lymph node dissection is longer than 20 cases, while in pancreatic cancer this curve is hard to be surpassed due to the low number of cases diagnosed with this pathology $y^{23,24}$.

More promising results in terms of sentinel lymph node dissection in pancreatic tumors came from Spain; this time the authors only referred to pancreatic head adenocarcinoma and included in their study seven such patients with presumed early stages of the disease. In all cases the mapping was performed after Technetium injection one day before surgery via endoscopic ultrasonography. At the time of surgery, resection with curative intent was performed in four cases, while the sentinel nodes were identified in two cases, in both cases the nodes being found at the level of the inter-aorto-cava region. However, the final histopathological studies demonstrate the presence of lymph node metastases into a single sentinel node ${ }^{25}$.

Another interesting study which focused on the role of sentinel node biopsy in pancreatic head adenocarcinoma came from the Japanese authors conducted by Ohta et al; the authors initially demonstrated that number 13 (posterior pancreatic-duodenal lymph node group) represent a major regional drainage site of the pancreatic head, the status of this nodes predicting that of the number 16 (abdominal para-aortic lymph node group ${ }^{26}$. In the current study, the authors injected $2 \%$ patent blue dye in the peritumoral area, and 5 minutes later, the blue stained nodes within the area of the no. 13 lymph node group were identified, excised and sent for the frozen section. The further surgical decision was based on the status of these nodes and consisted of extended lymph node dissection to number 16 lymph nodes if the sentinel nodes were positive and ending the procedure if the sentinel nodes at the level of the group number 13 were negatives. The authors proposed this protocol in 21 cases, nine of them being successfully submitted to this approach; among the nine cases, successful detection of the sentinel node at the level of the 
station number 13 was found in eight cases. Among these cases, there were four cases with positive sentinel nodes which were further submitted to extended lymph node dissection and four cases with negative sentinel nodes in which the surgical procedure was ended at this time. The overall 3-years survival rate for the entire group was $36 \%$, three of the four cases with negative sentinel nodes being alive at the three years follow-up. These data enabled the authors to declare that detecting the sentinel node in pancreatic head adenocarcinoma might provide an efficient identification of the patients at high risk, and might impede the surgeon to perform an unnecessary extended lymph node dissection ${ }^{27}$.

\section{Conclusions}

Data reported so far in cases with pancreatic adenocarcinomas are inconstant when it comes to the role of sentinel lymph node detection. This aspect is mainly related to the fact that most patients are diagnosed in advanced stages of the disease when the main lymphatic ducts are already obstructed by the tumoral involvement. Moreover, due to the relatively low number of cases in which resection with curative intent is feasible, the learning curve is very difficult to be surpassed. It seems that the best results in terms of sentinel lymph node detection are reported in cases presenting lower tumoral volumes as well as in those cases in which the tracer is injected in the peritumoral area and not directly in the center of the tumor. However, further studies are still needed in order to validate the method

\section{Compliance with Ethics Requirements:}

„The authors declare no conflict of interest regarding this article"

\section{References}

1. Diaconu C, Mateescu D, Balaceanu A, Marcu M, Jianu V, Stanica A. Pancreatic cancer presenting with paraneoplastic thrombophlebitis: a case report. Journal of Medicine and Life 2010;3(1): 96-99.

2. Siegel RL, Miller KD, Jemal A. Cancer statistics, 2015. CA Cancer J Clin. 2015; 65(1):5-29.

3. Katz MH, Hwang R, Fleming JB, Evans DB. Tumor-nodemetastasis staging of pancreatic adenocarcinoma. CA Cancer J Clin. 2008;58(2):111-125.

4. DiMagno EP, Reber HA, Tempero MA. AGA technical review on the epidemiology, diagnosis, and treatment of pancreatic ductal adenocarcinoma. American Gastroenterological Association. Gastroenterology. 1999;117(6):1464-1484.

5. Kedra B, Popiela T, Sierzega M, Precht A. Prognostic factors of long-term survival after resective procedures for pancreatic cancer. Hepatogastroenterology 2001; 48(42):1762-1766.
6. Nathanson SD, Shah R, Rosso K. Sentinel lymph node metastases in cancer: causes, detection and their role in disease progression. Semin Cell Dev Biol 2015;38:106-116.

7. Stacker SA, Williams SP, Karnezis T, Shayan R, Fox SB, Achen MG. Lymphangiogenesis and lymphatic vessel remodelling in cancer. Nat Rev Cancer. 2014;14(3):159-172.

8. Whipple AO. The rationale of radical surgery for cancer of the pancreas and ampullary region. Ann Surg. 1941;114(4):612-615.

9. Bhatti I, Peacock O, Awan AK, Semeraro D, Larvin M, Hall RI. Lymph node ratio versus number of affected lymph nodes as predictors of survival for resected pancreatic adenocarcinoma. World J Surg. 2010; 34(4):768-775.

10. Doi R, Kami K, Ito D, et al. Prognostic implication of para-aortic lymph node metastasis in resectable pancreatic cancer. World J Surg. 2007; 31(1):147-154.

11. Murakami Y, Uemura K, Sudo T, et al. Number of metastatic lymph nodes, but not lymph node ratio, is an independent prognostic factor after resection of pancreatic carcinoma. $J$ Am Coll Surg. 2010; 211(2):196-204.

12. Kobayashi K, Sadakari Y, Ohtsuka T, et al. Factors in intraductal papillary mucinous neoplasms of the pancreas predictive of lymph node metastasis. Pancreatology. 2010; 10(6):720-725.

13. Masui T, Kubota T, Aoki K, et al. Long-term survival after resection of pancreatic ductal adenocarcinoma with para-aortic lymph node metastasis: case report. World J Surg Oncol. 2013;11(1):195.

14. Peparini N. Mesopancreas: a boundless structure, namely the rationale for dissection of the paraaortic area in pancreaticoduodenectomy for pancreatic head carcinoma. World J Gastroenterol. 2015; 21(10):2865-2870.

15. Tol JA, Gouma DJ, Bassi C, et al. Definition of a standard lymphadenectomy in surgery for pancreatic ductal adenocarcinoma: a consensus statement by the International Study Group on Pancreatic Surgery (ISGPS). Surgery. 2014; 156(3):591-600.

16. Svoronos C, Tsoulfas G, Katsourakis A, Noussios G, Chatzitheoklitos E, Marakis NG. Role of extended lymphadenectomy in the treatment of pancreatic head adenocarcinoma: review and meta-analysis. ANZ J Surg. 2014;84(10):706-711.

17. Ke K, Chen W, Chen Y. Standard and extended lymphadenectomy for adenocarcinoma of the pancreatic head: a meta-analysis and systematic review. J Gastroenterol Hepatol. 2014;29(3):453-462.

18. Noji T, Narasaki H, Ebihara Y, et al. Near infrared fluorescence light imaging for lymphatic flow toward para-aortic lymph node from the Calot's triangle: a prospective exploratory study. Photodiagnosis Photodyn Ther. 2018;24:274-279.

19. Kitagawa H, Ohta T, Makino I H, et al. Carcinomas of the ventral and dorsal pancreas exhibit different patterns of lymphatic spread. Front Biosci 2008; 13:2728-2735.

20. Roche CJ, Hughes ML, Garvey CJ, et al. CT and pathologic assessment of prospective nodal staging in patients with ductal adenocarcinoma of the head of the pancreas. AJR Am J Roentgenol. 2003; 180(2):475-480.

21. Imai H, Doi R, Kanazawa H, et al. Preoperative assessment of para-aortic lymph node metastasis in patients with pancreatic cancer. Int J Clin Oncol. 2010; 15(3):294-300.

22. Hirono S, Tani M, Kawai M, et al. Identification of the lymphatic drainage pathways from the pancreatic head guided by indocyanine green fluorescence imaging during pancreaticoduodenectomy. Dig Surg. 2012; 29(2):132-139. 
23. Durczynski A, Hogendorf P, Szymanski D, Grzelak P, Strzelczyk J. Sentinel lymph node mapping in tumors of the pancreatic body: preliminary report. Contemp Oncol (Pozn). 2012; 16(3):206-209.

24. Hutchinson JR, Chagpar AB, Scoggins CR, et al. Surgeon and community factors affecting breast cancer sentinel lymph node biopsy. Am J Surg. 2005;190(6):903-906.
25. Beisani M, Roca I, Cardenas R, et al. Initial experience in sentinel lymph node detection in pancreatic cancer. Rev Esp Med Nucl Imagen Mol. 2016;35(5):287-291.

26. Kayahara M, Nagakawa T, Ohta T, et al. Analysis of paraaortic lymph node involvement in pancreatic carcinoma: a significant indication for surgery? Cancer. 1999; 85(3):583-590.

27. Ohta T, Kitagawa H, Kayahara M, et al. Sentinel lymph node navigation surgery for pancreatic head cancers. Oncol Rep. 2003; 10(2):315-319. 